\title{
Learning Object-level Impedance Control for Robust Grasping and Dexterous Manipulation
}

\author{
Miao $\mathrm{Li}^{1}$, Hang Yin ${ }^{1}$, Kenji Tahara ${ }^{2,1}$ and Aude Billard ${ }^{1}$
}

\begin{abstract}
Object-level impedance control is of great importance for object-centric tasks, such as robust grasping and dexterous manipulation. Despite the recent progress on this topic, how to specify the desired object impedance for a given task remains an open issue. In this paper, we decompose the object's impedance into two complementary componentsthe impedance for stable grasping and impedance for object manipulation. Then, we present a method to learn the desired object's manipulation impedance (stiffness) using data obtained from human demonstration. The approach is validated in two tasks, for robust grasping of a wine glass and for inserting a bulb, using the 16 degrees of freedom Allegro Hand mounted with the SynTouch tactile sensors.
\end{abstract}

\section{INTRODUCTION}

Robust grasping and dexterous manipulation are two of the most important capabilities that a robot is expected to have. The main characteristic of a robust grasp is its ability to comply with external perturbations applied to the grasped object while still maintaining the grasp. In dexterous manipulation, the robotic hand, mainly the fingertips, have to physically interact with the object in order to move it to a desired configuration. In both scenarios, appropriate grasping forces need to be applied on the grasped or manipulated object, either to keep the grasp stable under perturbation or to move the object to a desired configuration.

To this end, various control algorithms have been proposed and ported to control multi-fingered robotic hand. These can be roughly divided into two groups. The first group encompass hybrid position/force control approaches that modulates the force explicitly to manage the interaction imposed by the environment [1], [2], [3]. Another group uses impedance control to regulate the interaction force implicitly by specifying the impedance of the grasped object [4], [5], [6]. [7]. In general, the hybrid position/force control is more precise when controlling simultaneously the force and position. The main deficiency of hybrid control is the transition between position and force control when the contact state varies between noncontact and contact. A small delay in this transition may lead to a very large overshot contact force. In addition, the selection of accurate grasping forces for hybrid control that fulfil the friction constraints and task requirements is still a difficult planning problem [3]. In impedance controller, the object motion is realized by a desired object impedance that

\footnotetext{
${ }^{1}$ M. Li, H. Yin and A. Billard are with LASA, École Polytechnique Fédérale de Lausanne (EPFL), Switzerland \{miao.li, hang.yin, aude.billard\} @epfl.ch

${ }^{2} \mathrm{~K}$. Tahara is with Faculty of Engineering, Kyushu University, 744 Moto'oka, Nishi-ku, Fukuoka 819-0395, Japan. $\mathrm{He}$ is currently a visiting scholar at LASA. taharadieee.org, kenji.tahara@epfl.ch
}

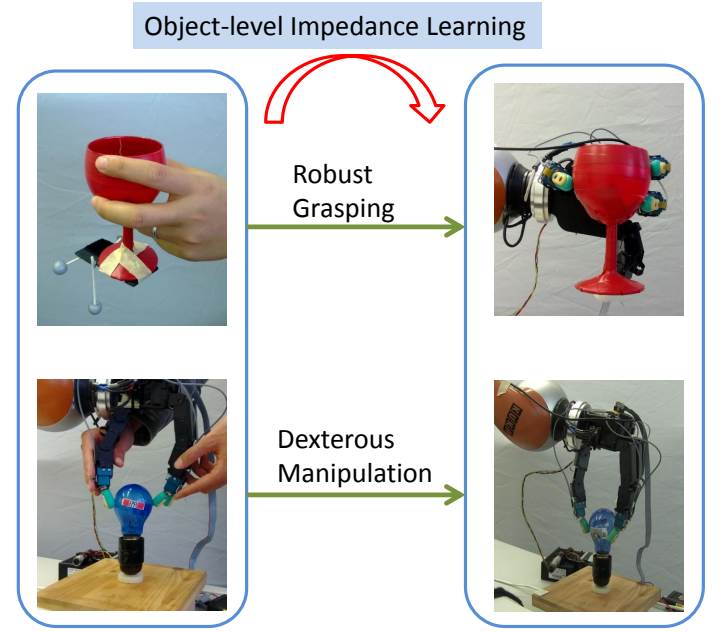

Fig. 1: The object-level impedance for robust grasping and dexterous manipulation are learned from human demonstration.

generates force to move the object to a desired configuration. It has the advantage that it will converge to the desired position in free motion and a stable equilibrium position in the case of interaction with the environment. This merit can be greatly beneficial for both robust grasping and dexterous manipulation. Therefore, we restrict the rest of this review to impedance controllers only. In [8], a fingertip Cartesian stiffness controller was introduced using fingertip force sensor. However, the stiffness controller can not actively control the whole system dynamics. To overcome this defect, Liu and Hirzinger [9] proposed a Cartesian impedance controller for the DLR hand based on the joint torque measurements. While these two controllers are in the fingertip Cartesian space, object-level impedance controllers are proposed by directly specifying the desired impedance in the object frame, which are usually more suitable for robust grasping and dexterous manipulation of an object. In [4], an object level impedance controller has been proposed for a multi-arm manipulator to directly control the internal object forces and compensate the system dynamics. The object is assumed to be rigidly grasped that can transmit bilateral contact forces between the fingertips and the object. Wimbock et al. [5], [7] recently presented their experimental evaluation of an intrinsically passive controller for multi-fingered hand, where the damping parameters are designed and implemented as a function of the object effective inertia and stiffness matrix. A similar impedance controller was also proposed in [6] and [10] by defining a virtual frame which depends only on the fingertip positions. The damping parameters are designed in 
both the finger joint space and the object frame.

However, despite all the above-mentioned progress, one critical issue still remains unaddressed: how to specify the proper impedance for a given task? The specification of impedance is known as a difficult problem as it depends on the task at hand as well as the kinematic and dynamic limitation of the robot [11], [12]. Moreover, the impedance parameters may need to adapt to the task requirements or to variation in the environment, such as the bulb replacement task that the torsional resistance increases greatly during the last phase of the task. To this end, sensor feedback should be taken into account to monitor the status of task's completion and to vary the impedance accordingly.

In this paper, we attempt to address this problem by learning the impedance from human demonstration. In the following Section II, some related works regarding impedance specification are summarized. In Section III, an object-level impedance controller is reformulated. In Section IV, methods for learning impedance from human demonstration for robust grasping and dexterous manipulation are presented. Experiments on a multi-fingered robotic hand are demonstrated and discussed in Section V. Finally, we give a conclusion and an outlook on future work in Section IV.

\section{RELATED WORK}

a) Analytical Impedance Specification: In one of their early works, Mason and Salisbury [8] used the congruence transformation to obtain the desired object stiffness from joint stiffness. Based on this work, Cutkosky and Kao [13] expressed the compliance of a grasp as a function of grasp geometry, contact conditions and mechanical properties of the fingers. In order to choose the grasp compliance for a given task, Shimoga and Goldenberg [14] formulated a concept termed Grasp Admittance Center, which is the origin of a frame that impedance matrices will be diagonal. Also, a qualitative method has been developed to choose the relative magnitude of the impedance parameters for a set of tasks. In [15], [16], Kim et al. analysed the compliance characteristic for different tasks by considering the grasp geometry, which is the relation between the operational space and the fingertip space of multi-fingered hand. Their analytical results show that the non-diagonal terms in impedance matrices can not be specified arbitrarily and they also used a qualitative method (small and large value of stiffness) to specify the impedance parameter.

b) Impedance Learning: Learning of tasks is another approach by which desired impedance parameters can be specified. In [17], the impedance learning problem is formulated as a model-based reinforcement learning problem, where the impedance parameters can gradually change to improve the task performance. In [18], the authors accomplished a variable impedance controller with a modelfree, sample-based reinforcement learning method. However, the reinforcement function needs to be carefully defined to capture the essence of the task, which will be difficult for complex tasks, such as dexterous manipulation. Sikka and McCarragher [19] presented a method that can learn

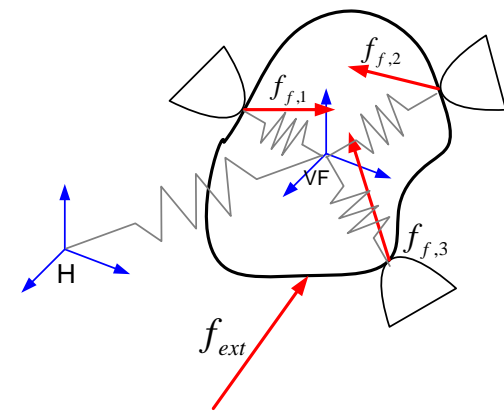

Fig. 2: An object grasped by 3 fingers. The object impedance and grasp impedance are shown as springs. The $\mathbf{f}_{f, i}, i=1,2,3$ are the contact forces on each fingertips. $\mathbf{f}_{e x t}$ is the external perturbation force. The frame $\mathbf{H}$ and VF are the inertial frame and the virtual frame, respectively.

the robot end-point stiffness of contact tasks from human demonstration. An online, incremental algorithm has been proposed in [20] to learn varying end-point stiffness from human demonstration. For a multi-fingered robotic hand, a implicit compliant controller [21] is learned to adapt the grasp under perturbation, which actually mapping the fingertips tactile response to finger joints. However, this method is hand dependent and difficult to generalize to manipulation tasks.

As discussed in [22], the tasks for multi-fingered hand are usually object-centric. In these cases, learning an objectlevel impedance is more suitable and can be easily applied to other hands. In this paper, we extend the object-level impedance controller in [10] with tactile feedback and objectlevel impedance learned from human demonstration.

\section{OBJECT-LEVEL IMPEDANCE CONTROL}

In this section, we will reformulate the object-level impedance controller proposed in [10], which is mainly composed of two parts, a stable grasp controller and an object manipulation controller.

\section{A. Object Manipulation Impedance}

Following the formulation of impedance control in [11], the dynamics of the object, as shown in Fig. 2, is governed by the equation:

$$
\mathbf{f}_{f, o}+\mathbf{f}_{e x t}=M_{0} \ddot{\mathbf{x}}
$$

where $\mathbf{f}_{f, o}$ is the summation of manipulating forces $\mathbf{f}_{f, o i}$ exerted on the object from each fingertip, $\mathbf{f}_{\text {ext }}$ is the external perturbation force. All the forces are expressed in the inertial frame. $M_{0}$ is the actual inertia matrix and $\mathrm{x}$ is the position and orientation of the object. Usually, the position and orientation are controlled independently [10]. Here for simplicity, we put position and orientation in one vector $\mathbf{x}$ to introduce the controller.

The objective of impedance control is to modulate the interaction between the object and the environment by controlling the contact forces. The desired interaction of the system is given by:

$$
\mathbf{f}_{e x t}=M \ddot{\mathbf{x}}+D\left(\dot{\mathbf{x}}-\dot{\mathbf{x}}_{r}\right)+K\left(\mathbf{x}-\mathbf{x}_{r}\right)
$$


where $\mathbf{x}_{r}, \dot{\mathbf{x}}_{r}$ is the reference trajectory and $M, D, K$ are the desired apparent inertia, damping and stiffness, respectively. From equations (1) and (2), we can derive the object-level impedance control law given as:

$$
\mathbf{f}_{f, o}=E D\left(\dot{\mathbf{x}}_{r}-\dot{\mathbf{x}}\right)+E K\left(\mathbf{x}_{r}-\mathbf{x}\right)+(E-I) \mathbf{f}_{e x t}
$$

where $E=M_{0} M^{-1}$ and $I$ is the identity matrix. In practice, it is often sufficient to keep the inertia unchanged, i.e., $M_{0}=M$ and only shape the stiffness and damping. Then the equation (3) can be simplified as:

$$
\mathbf{f}_{f, o}=D\left(\dot{\mathbf{x}}_{r}-\dot{\mathbf{x}}\right)+K\left(\mathbf{x}_{r}-\mathbf{x}\right)
$$

\section{B. Stable Grasping Impedance}

Up to now, only the object manipulation impedance has been considered. In order to make the grasp stable during manipulation, we need to design a stable grasping impedance, which can be used to change the grasping forces. In our paper, the contact model between the object and the fingertips is assumed to be point contact with friction, which can only transmit contact forces. Therefore, we only use one translational spring connecting each fingertip and the origin of the object (virtual) frame to represent the stable grasping impedance (stiffness), as shown in Fig. 2. The grasping forces can be expressed as:

$$
\mathbf{f}_{f, g i}=K_{g i}\left(\left\|\Delta \mathbf{p}_{i}\right\|-L_{i}\right) \frac{\Delta \mathbf{p}_{i}}{\left\|\Delta \mathbf{p}_{i}\right\|}
$$

where $\mathbf{f}_{f, g i}$ and $K_{g i}$ are the grasping force and stable grasping stiffness at $i$-th fingertip. $\Delta \mathbf{p}_{i}=\mathbf{p}_{o}-\mathbf{p}_{i}$ with $\mathbf{p}_{i}$ as the position of contact point on $i$-th fingertip and $\mathbf{p}_{o}$ as the position of the object frame origin. $L_{i}$ is the desired distance from the $i$-th fingertip to the object frame origin.

\section{Implementation Issues}

A rigorous implementation of the controller will require a lot of computational load [23]. To reduce it, the finger dynamics is not compensated and thus joint torques at each finger can be obtained from a simple Jacobian transpose.

$$
\boldsymbol{\tau}_{f, i}=J_{f, i}^{T} \mathbf{f}_{f, i}
$$

where $\boldsymbol{\tau}_{f, i}$ are the joint torques at $i$-th finger and $J_{f, i}$ is the Jacobian of the $i$-th finger. The contact force can be computed as: $\mathbf{f}_{f, i}=\mathbf{f}_{o, i}+\mathbf{f}_{g, i}$. The more rigorous way to compute the contact force using grasp mapping can be also use here [7], which is also more computational expensive.

In order to implement this controller, we need to address the following issues: (a) measure the object position and orientation $\mathbf{x}$; (b) design the reference trajectory $\mathbf{x}_{r}, \dot{\mathbf{x}}_{r}$; (c) choose the impedance parameters $K$ and $D$. While (a) will be discussed in the remaining part of this section by introducing a Virtual Frame, the method to deal with (b) and (c) by learning from human demonstration will be presented in the next section.

\section{Virtual Object Frame}

Due to the occlusion of the hand, it is still very difficult to rely on vision to obtain the actual object position and orientation in the controller. To deal with this, the concept of Virtual Frame (VF) is adopted here, which is a function of all the contact points between object and fingertips. Virtual frame (VF) can be used to estimate the real object position and orientation if we assume that relative contact points between object and fingertips do not change ${ }^{1}$. Different from the definition in [10], the VF in this work is the function of real contact point on each fingertip, which can be obtained from tactile feedback. In our work, we only use three fingers, the origin of VF is:

$$
\mathbf{p}_{o}=\frac{1}{3} \sum_{i=1}^{3} \mathbf{p}_{i}
$$

The orientation of the frame is defined in the following way:

$$
\begin{aligned}
& R_{o}=\left[\mathbf{r}_{x}, \mathbf{r}_{y}, \mathbf{r}_{z}\right] \in S O(3) \\
& \mathbf{r}_{x}=\frac{\mathbf{p}_{3}-\mathbf{p}_{1}}{\left\|\mathbf{p}_{3}-\mathbf{p}_{1}\right\|} \\
& \mathbf{r}_{z}=\frac{\left(\mathbf{p}_{2}-\mathbf{p}_{1}\right) \times \mathbf{r}_{x}}{\left\|\left(\mathbf{p}_{2}-\mathbf{p}_{1}\right) \times \mathbf{r}_{x}\right\|} \\
& \mathbf{r}_{y}=\mathbf{r}_{z} \times \mathbf{r}_{x}
\end{aligned}
$$

With the defined VF, one can compute the translation and rotation difference between the VF and the desired or reference frame. Thus, from equation (4), (5) and (6), the desired joint torque for each finger can be calculated. For more details about the implementation, one can refer to [10].

\section{IMPEDANCE LEARNING FROM HUMAN DEMONSTRATION}

In this section, methods about how to specify impedance for robust grasping and dexterous manipulation will be presented.

\section{A. Relative Impedance for Robust Grasping}

A robust grasp should be able to comply with external perturbation from any directions. But in different directions, the extent of compliance will depend on the grasp configuration as well as the task requirement. For instance, grasping a screwdriver as a tool and grasping a pen to write will require totally different levels of rotational compliance along the axial direction.

Our method of impedance selection for robust grasping is quite intuitive: the object stiffness in one direction is inversely proportional to the variance of displacement under perturbation in the corresponding direction. From this assumption, we can learn the relative stiffness for robust grasping in different directions from human demonstration. This idea has also been utilized to learn the end-point stiffness for a single manipulator [20]. During the demonstration, an object is grasped by a human demonstrator with eyes closed, to mimic the fact that our controller will use solely

\footnotetext{
${ }^{1}$ This assumption will neglect the rolling and slipping effects.
} 
proprioceptive and tactile information, with no vision. The grasped object is perturbed by another person randomly and the displacement of the object is recorded $\left\{\mathrm{x}^{i}, i=1 \ldots N\right\}$. Then the object stiffness can be specified as follows:

$$
K=\alpha\left\{\frac{1}{N} \sum_{i=1}^{N}\left(\mathbf{x}^{i}-\mathbf{x}_{r}\right)\left(\mathbf{x}^{i}-\mathbf{x}_{r}\right)^{T}\right\}^{-1}
$$

where $\alpha \in \mathbb{R}^{+}$is a ratio parameter that needs to be set manually and $\mathbf{x}_{r} \in \mathbb{R}^{6}$ is the object initial (and desired) position and orientation.

Besides stiffness specification, object workspace modelling is also very important for robust grasping as it determines the extent of motion of a grasped object during perturbation. However, the workspace of a grasped object will depend on the hand kinematics and the grasp configuration. To this end, we teach the robot the extend to which it can stretch its fingers through kinaesthetic demonstration, by back-driving the fingers, see Fig. 3. All positions and orientations adopted by the hand during the demonstration are used to build a probabilistic model of the workspace of the hand. The use of a probabilistic model is advantageous as it accounts for the imprecision of the recording and allows to generalize outside the demonstrations. The latter is particularly important since demonstrations may not be exhaustive and may not explore all possible postures. Here, we use Gaussian Mixture Model (GMM). A GMM is a probabilistic model of density function composed of $K$ Gaussian components. The likelihood of each position/orientation under this model is given by:

$$
p(\mathbf{x})=\sum_{k=1}^{K} \pi_{k} \mathcal{N}\left(\mathbf{x} \mid \boldsymbol{\mu}_{k}, \boldsymbol{\Sigma}_{k}\right)
$$

where $\pi_{k}$ is the prior of the $k$ th Gaussian component and $\mathcal{N}\left(\boldsymbol{\mu}_{k}, \boldsymbol{\Sigma}_{k}\right)$ is the Gaussian distribution with mean $\boldsymbol{\mu}_{k}$ and covariance $\boldsymbol{\Sigma}_{k}$. A new VF, computed from (7) and (8), is said to lie in the object's workspace if its likelihood belongs to the model is greater than a fixed threshold, i.e. $p\left(x_{*}\right)>L_{\text {thresh }}$. This threshold, in our experiment, is quite conservative and is set as no more than 2 standard deviation, which means that about $95.45 \%$ training position/orientation of VF will be covered by the learned GMM. For more details about the parameters selection for training GMM, one can refer to [24].

With the object workspace model, one can design a desired reaction behavior to improve grasping stability, e.g., increase object stiffness gradually when the object is approaching boundary of learned working space. This could be achieved in our model by increasing the ratio parameter $\alpha$ in equation (9). This would however increase stiffness by the same amount in all directions. It may often be useful to be able to shape this increase along particular directions, such as the direction the moves the object farthest away from the workspace's boundary.

\section{B. Variable Impedance for Dexterous Manipulation}

In the case of robust grasping, the reference frame can be easily set as the initial position and orientation, which

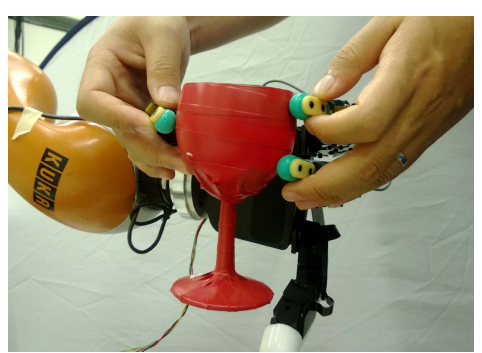

(a)

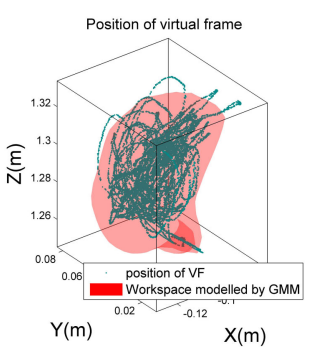

(b)
Fig. 3: (a) Human teaching of object workspace. The object impedance are set to zero in all directions during the demonstration, which means human can move the object freely in its workspace. (b) The position and orientation of VF are recorded and trained using GMM. Here is shown the trained result in the subspace of VF position. The red surface is the iso-surface with the same threshold likelihood $L_{\text {thresh }}$

does not vary with time. For dexterous manipulation, a timevarying reference trajectory $\mathbf{x}_{r}, \dot{\mathbf{x}}_{r}$ will be required. In this section, we will present a method that learns the reference trajectory and the desired object impedance simultaneously.

The objective of human demonstration is to model the interaction between the object being manipulated and the environment. Thus, during the demonstration, at each sample instant $i, i=1 \ldots N_{s}$, the motion of the object $\{\mathbf{x}(i), \dot{\mathbf{x}}(i)\}$ and the sum of manipulating forces $\mathbf{f}_{f, o}(i)$ applied on the object are recorded ${ }^{2}$. Consider $t=1 \ldots N_{t}$ consecutive samples of data obtained over a short time window. Assuming the impedance parameters and reference trajectory remain constant over this time window, the relationship between the object motion and the force exerted on object is given by:

$$
\mathbf{f}_{f, o}(i)=D\left(\dot{\mathbf{x}}_{r}-\dot{\mathbf{x}}(i)\right)+K\left(\mathbf{x}_{r}-\mathbf{x}(i)\right), i=1 \ldots N_{t} ;
$$

During each time window, since we assume that the object's impedance parameters and the reference trajectory are not changing with time, they can be obtained by minimizing the following objective function:

$$
\min _{D, K, \dot{\mathbf{x}}_{r}, \mathbf{x}_{r}} \sum_{i=1}^{N_{t}}\left\|\mathbf{f}_{f, o}(i)-\left\{D\left(\dot{\mathbf{x}}_{r}-\dot{\mathbf{x}}(i)\right)+K\left(\mathbf{x}_{r}-\mathbf{x}(i)\right)\right\}\right\|^{2}
$$

In practice, the term from damping is usually ignored by assuming that the desired velocity trajectory is the same as the measured one and thus equation (12) can be simplified as:

$$
\min _{K, \mathbf{x}_{r}} \sum_{i=1}^{N_{t}}\left\|\mathbf{f}_{f, o}(i)-\left\{K\left(\mathbf{x}_{r}-\mathbf{x}(i)\right)\right\}\right\|^{2}
$$

Note that the assumption that the object's impedance parameters and reference trajectory are stationary for short periods of time is only valid for tasks with small motion and low velocities, where the dynamics is mainly dominated by the compliance and contact condition. For fast tasks, it will require some high speed (force and motion) sensors to collected enough data points to obtain a reasonable

\footnotetext{
${ }^{2}$ In practice, only the contact forces on each fingertip can be measured, which include the grasping forces and the manipulating forces. the sum of grasping forces is very small in our setting from (5), which can be ignored.
} 
impedance estimation. Also, from (13), we can obtain the desired impedance parameters for each time window and their corresponding reference trajectory. In this framework, the desired impedance parameters and the reference trajectory will depend on time. To account for this, we define a variable $\phi \in[0,1]$ to represent the completion of the task, which is a function of the desired trajectory, i.e., $\phi=\Phi\left(\mathbf{x}_{r}\right)$. In our experiments, $\Phi$ is given by the distance from the current configuration to the goal configuration. Thus, the impedance parameters and desired trajectory are expressed as a function of $\phi$.

Since the system should be stable, additional constraints should be taken into account. First, the stiffness matrix should be positive semi-definite and its elements must be less than some maximum value since we assume that human will not demonstrate extremely large object stiffness. Also, for the reference trajectory, it should not be too far away from the actual measured object trajectory. Thus we have:

$$
\begin{aligned}
& K_{i, j} \leq k_{l i m}, \quad i=1 \ldots 6, j=1 \ldots 6 \\
& \left.\| \mathbf{x}_{r}-\mathbf{x}(i)\right) \| \leq \Delta x_{l i m}, \quad i=1 \ldots N_{t} \\
& \left\|\dot{\mathbf{x}}_{r}-\dot{\mathbf{x}}(i)\right\| \leq \Delta \dot{x}_{l i m}, \quad i=1 \ldots N_{t}
\end{aligned}
$$

where $\Delta x_{\text {lim }} \in \mathbb{R}^{+}, \Delta \dot{x}_{\text {lim }} \in \mathbb{R}^{+}$is upper bound of the difference between the actual and real (position and velocity) trajectories. With the objective function (13) and the constraints (14), the MATLAB built-in constrained optimization function fmincon is used to find the optimized impedance parameters and reference trajectory.

\section{EXPERIMENTS AND DISCUSSION}

In the experiments, we use a 4 -fingered Allegro hand ${ }^{3}$ to test the object impedance specification for robust grasping and dexterous manipulation. The initial grasp and the grasping stiffness are predefined.

\section{A. Setup}

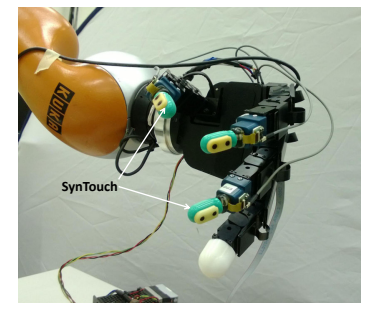

(a) Allegro hand

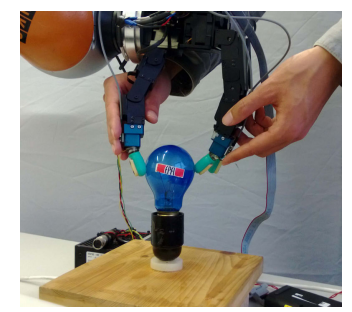

(b) Human demonstration
Fig. 4: (a) The Allegro hand mounted with the SynTouch tactile sensors on the fingertips; (b) Human demonstration of bulb replacement.

Each of the four fingers of the Allegro hand has 4 independent torque-controlled joints, see Fig. 4a. In our experiments, we only use 3 fingers even though our controller can be generalized to 4 fingers. Each fingertip of these 3 fingers has been mounted with a biometric tactile sensor from SynTouch ${ }^{4}$, which has been calibrated to provide contact information such as contact position and contact force.

\footnotetext{
${ }^{3}$ http://www.simlab.co.kr/Allegro-Hand.htm

${ }^{4}$ http://www.syntouchllc.com/
}

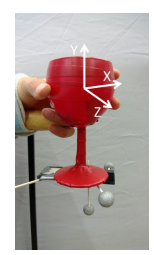

(a)

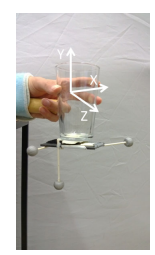

(b)

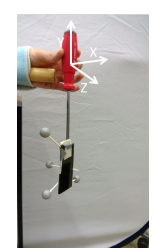

(c)

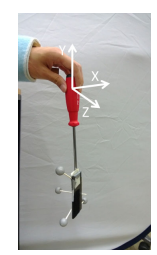

(d)
Fig. 5: Human demonstration of robust grasping on 3 different objects: glass, cup, screwdriver (side and top grasp). The motion of the object when perturbed is tracked by OptiTrack.

\section{B. Robust Grasping}

In the robust grasping experiment, a human expert demonstrates 4 grasps as shown in Fig. 5. The arm and wrist are fixated on the table so that the object motion will only come from the finger motion. During the experiments, for each object the perturbations are applied by another person randomly. The position and orientation for the objects are tracked using a motion capture system from OptiTrack ${ }^{5}$ at a sampling rate of $240 \mathrm{~Hz}$. More than 10000 datapoints are collected for each object.

The recorded object orientation is transformed into RPY Euler angles. The relative impedance parameters for the 4 grasps in different directions are computed using (9). In general, the choice of frame of reference depends on the task. Here we compute a diagonal stiffness matrix in the reference frame of the object since this is also the frame of reference in our impedance controller. It is also possible to extract the principle directions and corresponding stiffness along these directions from (9) [20], but then we need to transform the stiffness along these principle directions into the object's frame of reference in real time during implementation.

The relative stiffness for these grasps are shown in Fig. 6Fig. 9. The results indicate that in different directions, the relative stiffness for robust grasping is indeed different due to various task-specific requirements and grasps. This result may also imply that the anisotropic stiffness characteristic is optimal for robust grasping, in the sense of keeping the object stable with minimum efforts (grasping force). More experiments will be conducted in our future work to verify this implication.

Also, comparing the relative stiffness for two different grasps on the screwdriver (Fig. 5, (c) and (d)), see Fig. 8 and Fig. 9, we found that the rotational stiffness around $Y$-axis is totally different. In the top grasp, the rotational stiffness around $Y$-axis is much smaller than that of side grasp, which means that the top grasp requires smaller forces to rotate the screwdriver around $Y$-axis. This result also coincides with our intuition. Here, we only show the implementation results for the grasp on the glass. The parameters are set as follows: $K_{g i}=20 \mathrm{~N} / m, L_{i}=0.5\left\|\Delta \mathbf{p}_{i}\right\| m, k_{t x}=20 \mathrm{~N} / \mathrm{m}$, $k_{t y}=240 \mathrm{~N} / \mathrm{m}, k_{t z}=30 \mathrm{~N} / \mathrm{m}, k_{r x}=1.2 \times 10^{-3} \mathrm{Nm} / \mathrm{deg}$, $k_{r y}=6 \times 10^{-3} \mathrm{Nm} / \mathrm{deg}, k_{t z}=1.2 \times 10^{-3} \mathrm{Nm} / \mathrm{deg}$. The snapshot of the implementation on the Allegro hand is shown in Fig. 10.

${ }^{5}$ http://www.naturalpoint.com/optitrack/ 


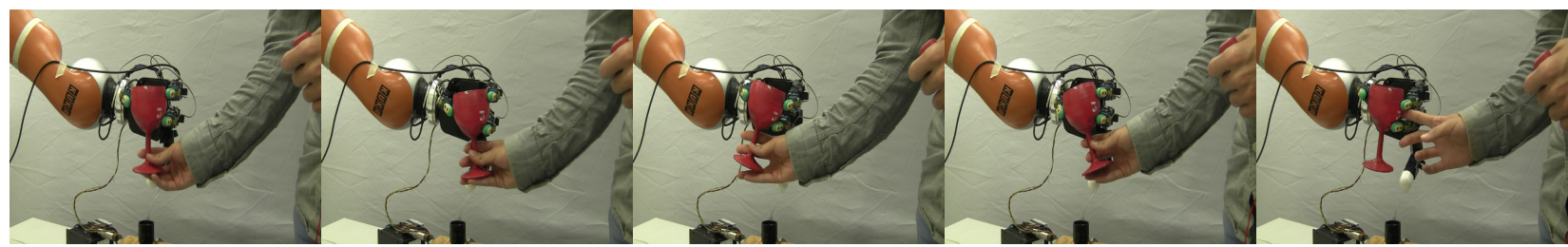

(a)

(b)

(c)

(d)

(e)

Fig. 10: Testing of robust grasping: Snapshots of the response of our controller when a human perturbs the original position of the glass. The fingers adapt smoothly to follow the direction of motion induced by the human. The impedance was learned from former human demonstration, using results in Fig. 6. The video is available at: http://lasa.epfl.ch/ miao/robust_grasping.wmv

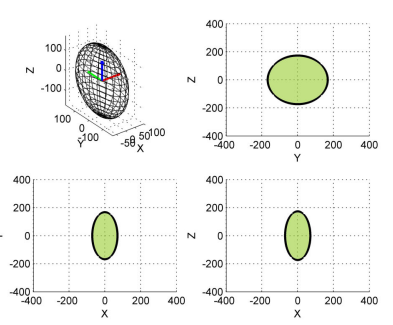

(a) glass

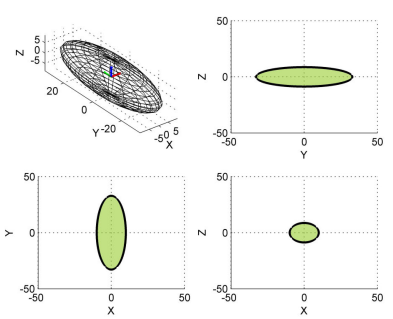

(b) glass

Fig. 6: (a): The relative translational stiffness for glass, $k_{t x}<k_{t z}<k_{t y}$; (b): The relative rotational stiffness for glass, $k_{r x} \approx k_{r z}<k_{t y}$.

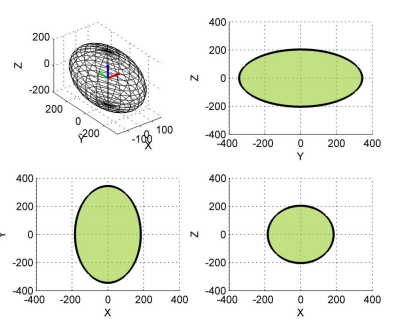

(a) cup

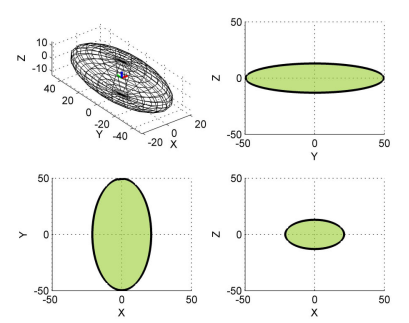

(b) cup

Fig. 7: (a): The relative translational stiffness for cup, $k_{t x} \approx k_{t z}<k_{t y}$; (b): The relative rotational stiffness for cup, $k_{r x}<k_{r z}<k_{t y}$.

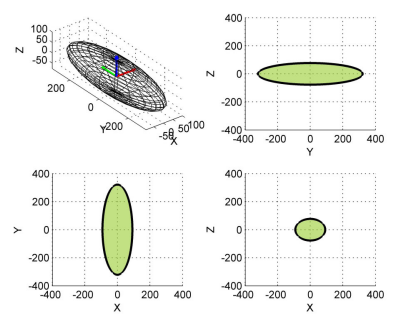

(a) screwdriver(side)

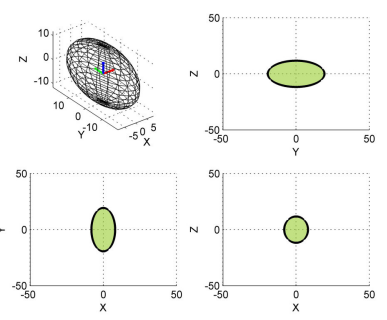

(b) screwdriver(side)

Fig. 8: (a): The relative translational stiffness for screwdriver (side grasp), $k_{t x} \approx k_{t z}<k_{t y} ;(\mathbf{b})$ : The relative rotational stiffness for screwdriver (side grasp), $k_{r x} \approx k_{r z}<k_{t y}$.

\section{Dexterous Manipulation}

For dexterous manipulation, we use the bulb replacement as an example, Fig. 4b. The bulb is initially on the socket already. During the human demonstration, only two fingers are used as the impedance is learned in object's frame of reference and using two fingers is easier to demonstrate. The manipulating forces are measured using SynTouch mounted on the fingertips. The object real trajectory is tracked using

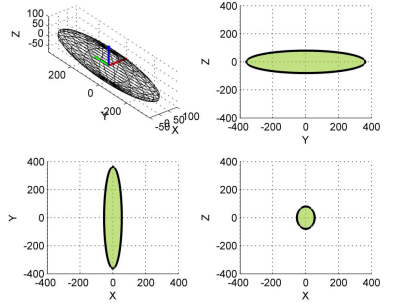

(a) screwdriver(top)
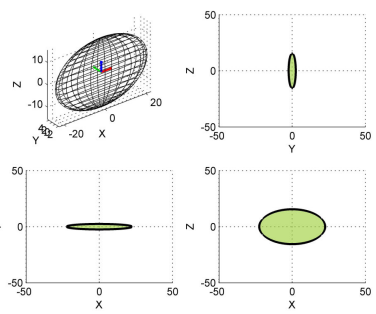

(b) screwdriver(top)
Fig. 9: (a): The relative translational stiffness for screwdriver (top grasp), $k_{t x} \approx k_{t z}<k_{t y}$; (b): The relative rotational stiffness for screwdriver (top grasp), $k_{t y}<k_{r x} \approx k_{r z}$.

OptiTrack ${ }^{6}$. Using equations (13) and (14), with $\Delta x_{\text {lim }}=$ $60 \mathrm{deg}, k_{\text {lim }}=100 \mathrm{~N} . \mathrm{mm} / \mathrm{deg}^{7}$, the reference trajectory and desired stiffness for bulb replacement are obtained and shown in Fig. 11 and Fig. 12a, respectively.

If we compare the desired rotation angle with the actual rotation angle, we see that the difference varies during the whole task. This means that human demonstrator indeed regulates the difference between the actual and reference trajectories as well as the stiffness parameter. When looking at the desired object stiffness, Fig. 12a, we see that the desired stiffness increases significantly during the last phase of the task. This is due to the fact that the resistance torque between the bulb and the socket increases significantly at the last phase. We repeated this demonstration 10 times, the obtained desired stiffness for each trial is shown in Fig. 12b.

If we could measure the rotational angle of the bulb using vision, then the status of task completion $\phi$ and the corresponding $k$ and $\mathbf{x}_{r}$ can be obtained directly, which will be very straightforward to implement the learned controller. Unfortunately, it is difficult to rely on vision for dexterous manipulation task as the hand often obstructs the object from the camera's view. For this reason, we rely on tactile information to guide the task process. Figure 12 indicates that the regulation of stiffness during this task follows two distinct phases. During the first phase, which occurs before break point $\phi=0.8$ (i.e. more than $2 / 3$ rd of the total duration of the task), the stiffness is quasi constant. Whereas in the second phase, it increases steadily. We model this

\footnotetext{
${ }^{6}$ During the human demonstration, in order to track the object robustly, the experimenter must take care of not placing his fingertips on the vision markers.

${ }^{7} \Delta x_{\text {lim }}$ is chosen by considering the rotation limitation of human hand and the Allegro hand.
} 
by setting a constant value for the stiffness for the first phase and by increasing linearly the stiffness up to its upper bound for the remainder of the task, i.e., $4 \mathrm{~N} . \mathrm{mm} / \mathrm{deg}$, see Fig. 12b. We noticed during the implementation that this breakpoint corresponds to the instant when one fingertip (usually the thumb of Allegro hand) starts slipping on the bulb. In order to detect the slippage, we use the contact forces $\mathbf{f}_{c}=\left[f_{c x}, f_{c y}, f_{c z}\right]$ from SynTouch on each fingertip, with $f_{c x}, f_{c y}$ and $f_{c z}$ being the tangential forces in two directions and the normal force, respectively. A slippage occurs at one fingertip if the contact forces that fingertip satisfying $\sqrt{f_{c x}^{2}+f_{c y}^{2}}>\mu f_{c z}, \mu$ is the coefficient of friction that is set manually. In our task, we choose $\mu=0.9$. The resulting control strategy is given in Algorithm 1. The snapshot of the implementation on Allegro hand is shown in Fig. 13.

Note that the current implementation of the bulb turning task is incomplete since the task status can not be robustly monitored only from tactile sensing. To ensure that the bulb is actually moving and that the fingers are not simply slipping on the object, vision should be used in conjunction with tactile information.

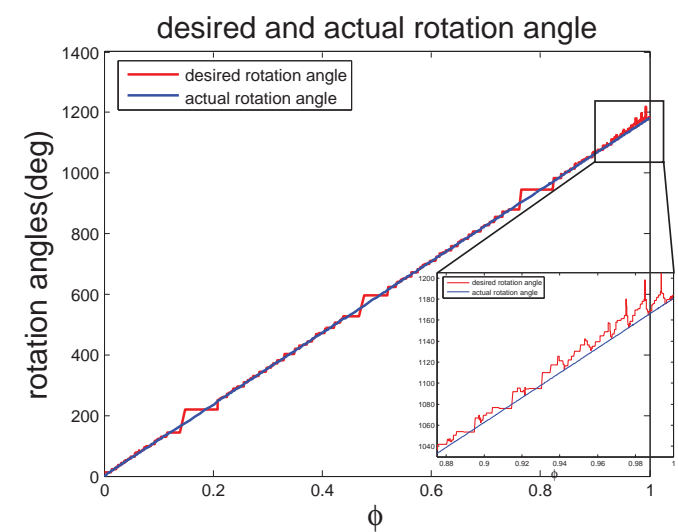

Fig. 11: The learned reference trajectory for trial $5 . \phi$ is the variable that represents the status of completion of the task, which is chosen as the ratio between current rotational angle and maximal rotational angle.

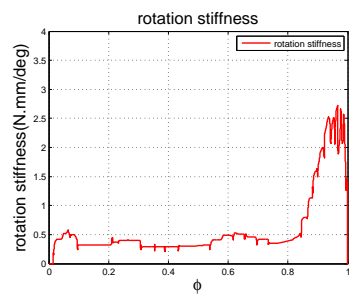

(a)

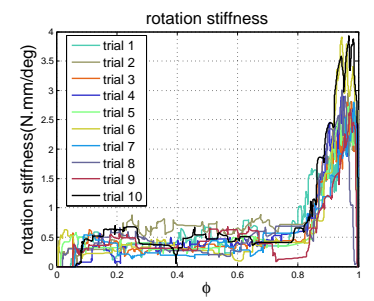

(b)
Fig. 12: (a) The learned desired object stiffness for trial 5 . The stiffness will significantly increase at the last phase of bulb replacement. (b) The learned desired object stiffness for 10 different trials.

\section{Discussion}

During the robust grasping, we didn't consider the problem of grasp stability. As studied in [25], the object dynamic stability will be closely related to the choice of grasp stiffness. In future work, we will investigate ways in which

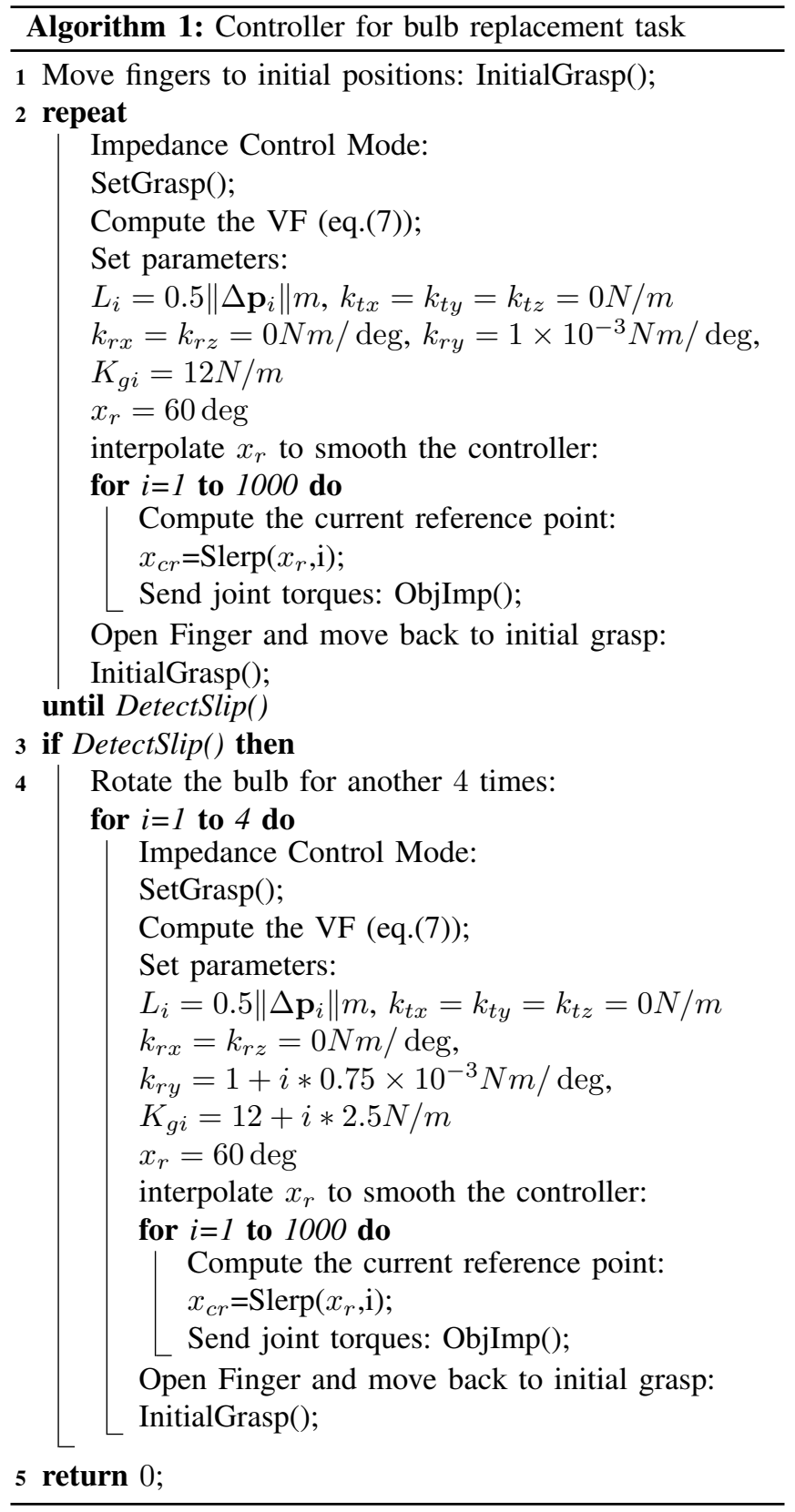

to shape the stiffness while taking the grasp stability into account.

Second, currently the initial grasp and grasp stiffness are predefined in our experiments, which is based on the assumption that the given grasp can realize the desired object impedance. However, given the object impedance specification and a multi-fingered robotic hand, how to choose a grasp that can realize this desired impedance will be a challenging extension direction. One of the possible ways will be employing the optimization framework for grasp synthesis in our previous work [26].

Furthermore, in our extended work, more tasks will be incorporated to demonstrate the generality of anisotropic stiffness in robust grasping and varying stiffness in dexterous manipulation. 


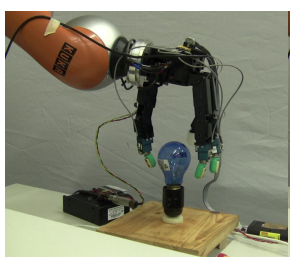

(a)

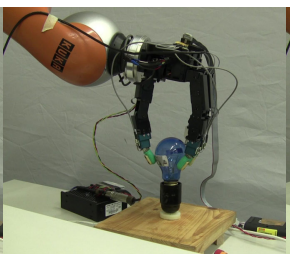

(b)

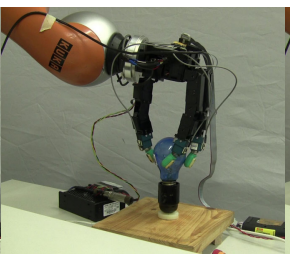

(c)

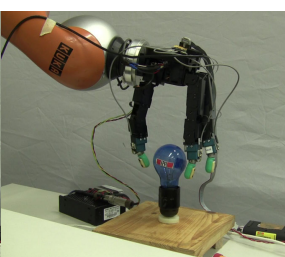

(d)

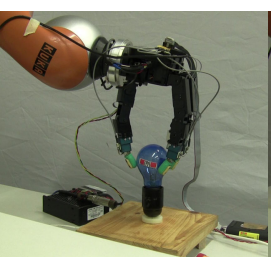

(e)

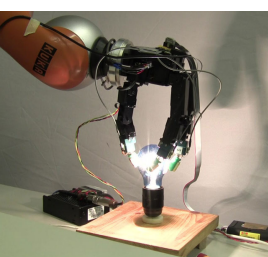

(f)

Fig. 13: The snapshots for dexterous manipulation. The video for this demo is available at: http://lasa.epfl.ch/ miao/bulb_replace.wmv

\section{CONCLUSIONS}

In this paper, an object-level impedance learning approach was proposed for both robust grasping and dexterous manipulation. For robust grasping, the relative stiffness is specified by measuring the displacement of object under perturbation. For dexterous manipulation, the desired reference trajectory and the desired object impedance is learned through an optimization-based approach. Both of these approaches are validated on a multi-fingered robotic hand. The results show that learning from human demonstration is an effective way to specify the desired impedance for object-centric tasks. We are currently working on integrating tactile feedback into the object impedance controller for grasping stiffness specification.

\section{ACKNOWLEDGMENT}

Miao Li was supported by the European Union Seventh Framework Programme FP7/2007-2013 under grant agreement $\mathrm{n}^{\circ} 288533$ ROBOHOW.COG. Hang Yin was supported partly by a FCT doctoral grant (SFRH/BD/51933/2012) under the IST-EPFL Joint Doctoral Initiative and by the Swiss National Center of Robotics Research. Kenji Tahara was supported by JSPS Grant-in-Aid for Young Scientists (A) (25700028).

\section{REFERENCES}

[1] Z. Li, P. Hsu, and S. Sastry, "Grasping and coordinated manipulation by a multifingered robot hand.," The International Journal of Robotics Research, vol. 8, no. 4, pp. 33-50, 1989.

[2] T. Yoshikawa and X.-Z. Zheng, "Coordinated dynamic hybrid position/force control for multiple robot manipulators handling one constrained object," The International Journal of Robotics Research, vol. 12, no. 3, pp. 219-230, 1993.

[3] Z. Li, Z. Qin, S. Jiang, and L. Han, "Coordinated motion generation and real-time grasping force control for multifingered manipulation," in Proceedings of International Conference on Robotics and Automation (ICRA), 1998.

[4] S. A. Schneider and R. H. Cannon, "Object impedance control for cooperative manipulation: theory and experimental results," IEEE Transactions on Robotics and Automation, vol. 8, no. 3, pp. 383-394, 1992.

[5] T. Wimbock, C. Ott, and G. Hirzinger, "Analysis and experimental evaluation of the intrinsically passive controller (IPC) for multifingered hands," in Proceedings of International Conference on Robotics and Automation (ICRA), 2008.

[6] K. Tahara, S. Arimoto, and M. Yoshida, "Dynamic object manipulation using a virtual frame by a triple soft-fingered robotic hand," in Proceedings of International Conference on Robotics and Automation (ICRA), 2010.

[7] T. Wimbck, C. Ott, A. Albu-Schffer, and G. Hirzinger, "Comparison of object-level grasp controllers for dynamic dexterous manipulation," The International Journal of Robotics Research, vol. 31, no. 1, pp. 323, 2012.
[8] M. T. Mason and J. K. Salisbury, Robot Hands and the Mechanics of Manipulation. The MIT series in Artificial Intelligence, Cambridge, Massachusetts: The MIT Press, 1985.

[9] H. Liu and G. Hirzinger, "Cartesian impedance control for the DLR hand," in Proceedings of International Conference on Intelligent Robots and Systems (IROS), 1999.

[10] K. Tahara, K. Maruta, A. Kawamura, and M. Yamamoto, "Externally sensorless dynamic regrasping and manipulation by a triple-fingered robotic hand with torsional fingertip joints," in Proceedings of International Conference on Robotics and Automation (ICRA), 2012.

[11] N. Hogan, "Impedance control - an approach to manipulation. i theory. II - implementation. III - applications," ASME Transactions Journal of Dynamic Systems and Measurement Control B, vol. 107, pp. 1-24, Mar. 1985.

[12] B. Siciliano, L. Sciavicco, L. Villani, and G. Oriolo, Robotics: Modelling, Planning and Control. Springer Publishing Company, Incorporated, 1st ed., 2008.

[13] M. Cutkosky and I. Kao, "Computing and controlling compliance of a robotic hand," IEEE Transactions on Robotics and Automation, vol. 5, no. 2, pp. 151-165, 1989.

[14] K. Shimoga and A. Goldenberg, "Grasp admittance center: Choosing admittance center parameters," in American Control Conference, 1991, pp. 2527-2532, 1991

[15] B.-H. Kim, B.-J. Yi, S.-R. Oh, and I. H. Suh, "Task-based compliance planning for multifingered hands," in Proceedings of International Conference on Robotics and Automation (ICRA), 2001

[16] B.-H. Kim, B.-J. Yi, S.-R. Oh, and I. H. Suh, "Fundamentals and analysis of compliance characteristics for multifingered hands," in Proceedings of International Conference on Robotics and Automation (ICRA), 2001

[17] B.-H. Yang and H. Asada, "Progressive learning and its application to robot impedance learning," IEEE Transactions on Neural Networks, vol. 7, no. 4, pp. 941-952, 1996.

[18] J. Buchli, F. Stulp, E. Theodorou, and S. Schaal, "Learning variable impedance control," The International Journal of Robotics Research, vol. 30, no. 7, pp. 820-833, 2011.

[19] P. Sikka and B. J. McCarragher, "Stiffness-based understanding and modeling of contact tasks by human demonstration," in Proceedings of International Conference on Intelligent Robots and Systems (IROS), 1997.

[20] K. Kronander and A. Billard, "Online learning of varying stiffness through physical human-robot interaction," in Proceedings of International Conference on Robotics and Automation (ICRA), 2012.

[21] E. L. Sauser, B. Argall, G. Metta, and A. Billard, "Iterative learning of grasp adaptation through human corrections.," Robotics and Autonomous Systems, vol. 60, no. 1, pp. 55-71, 2011.

[22] A. Okamura, N. Smaby, and M. Cutkosky, "An overview of dexterous manipulation," in Proceedings of International Conference on Robotics and Automation (ICRA), 2000.

[23] T. Yoshikawa, "Multifingered robot hands: Control for grasping and manipulation," Annual Reviews in Control, vol. 34, no. 2, pp. $199-$ 208, 2010.

[24] B. Huang, S. El-Khoury, M. Li, J. J. Bryson, and A. Billard, "Learning a real time grasping strategy," in Proceedings of International Conference on Robotics and Automation (ICRA), 2012.

[25] C.-H. Xiong, Y.-F. Li, H. Ding, and Y.-L. Xiong, "On the dynamic stability of grasping," The International Journal of Robotics Research, vol. 18, no. 9, pp. 951-958, 1999.

[26] S. El Khoury, M. Li, and A. Billard, "Bridging the gap: One shot grasp synthesis approach," in Proceedings of International Conference on Intelligent Robots and Systems (IROS), 2012. 